\title{
Magnitude of exercise capacity and quality of life improvement following repeat pulmonary rehabilitation in patients with COPD
}

This article was published in the following Dove Press journal:

International Journal of COPD

6 April 2017

Number of times this article has been viewed

\author{
Jacqueline S Sandoz $z^{1,2}$ \\ Mary M Roberts ${ }^{1,3,4}$ \\ Jin-Gun Cho',3-5 \\ John R Wheatley ${ }^{1,3-5}$ \\ 'Respiratory Ambulatory Care \\ Service, Western Sydney Local \\ Health District, NSW, Australia; \\ ${ }^{2}$ Canadian Alternatives in Non-invasive \\ Ventilation (CANVent) Program, \\ Ottawa Hospital Rehabilitation \\ Centre, Division of Respiratory \\ Medicine, Ottawa Hospital, Ontario, \\ Canada; ${ }^{3}$ Department of Respiratory \\ and Sleep Medicine, Westmead \\ Hospital, ${ }^{4}$ Ludwig Engel Centre for \\ Respiratory Research, Westmead \\ Institute for Medical Research, \\ ${ }^{5}$ Sydney Medical School, University of \\ Sydney, Westmead, NSW, Australia
}

Background: Maintenance and repeated pulmonary rehabilitation programs (PRPs) for patients with COPD have attempted to prolong PRP benefits beyond 12-24 months. However, there is limited evidence as to the magnitude of benefit or the ideal interval between repeating the program under "real-world" conditions in which patients are referred based on clinical necessity. Therefore, we reviewed the effects of repeating PRP in a physician-referred cohort of patients with COPD.

Methods: A total of 141 individuals with COPD completed PRP twice and 35 completed PRP three times over a 12-year period. We used linear mixed-effects models to quantify the magnitude and change in 6-minute walk distance (6MWD), St George's Respiratory Questionnaire (SGRQ), and Hospital Anxiety and Depression Scale (HADS) for each PRP. One-way analysis of variance with Tukey's post hoc analysis compared the effects of different time intervals on 6MWD, SGRQ, and HADS between PRPs.

Results: Despite $39 \mathrm{~mL} /$ year average decrease in forced expiratory volume in 1 second, overall 6MWD improved following each PRP (PRP1=58 m, $P<0.0001$; PRP2=42 m, $P<0.0001$; PRP3=32 m, $P<0.003)$. Mean SGRQ decreased after PRP1 $(-7.0$ units; $P<0.001)$ and PRP2 ( -4.9 units; $P<0.0001)$ but not after PRP3 ( -3.2 units; $P=0.10)$. HADS decreased after PRP1 $(-1.9$ units; $P<0.0001)$ and PRP2 $(-1.7$ units; $P=0.0001)$ but not after PRP3 $(-0.4$ units; $P=0.63)$.

Conclusion: In physician-referred patients who underwent repeat PRP as clinically required, there were clear benefits in functional exercise capacity following each repeat PRP, which was not affected by the time interval between PRPs. Health-related quality of life and mood improved after the first two PRPs, but not after a third.

Keywords: exercise therapy, chronic obstructive pulmonary disease, quality of life, exercise test

\section{Plain language summary}

Patients with chronic obstructive pulmonary disease (COPD) may improve their exercise ability and quality of life after a course of pulmonary rehabilitation. However, these benefits are lost over time, usually by $12-24$ months. The best time to repeat pulmonary rehabilitation and how much benefit can be gained from repeating rehabilitation are not well known. We studied 141 patients with COPD referred by physicians for at least two pulmonary rehabilitation programs (PRPs) over a 12-year period. Thirty-five of these patients completed the program three times. We found that exercise capacity improved after each PRPs, but there was less improvement after repeat PRPs. Quality of life and mood improved after the first two programs, but not after a third. Overall, there are clear benefits to patients with COPD repeating pulmonary rehabilitation as clinically required, which is not affected by the time interval between programs.
Correspondence: Jin-Gun Cho Department of Respiratory and Sleep Medicine, Westmead Hospital, PO Box 533, Wentworthville, NSW 2145, Australia Email jin.cho@sydney.edu.au 


\section{Introduction}

COPD is a leading cause of morbidity and mortality, with an increasing burden of disease worldwide. Pulmonary rehabilitation is a key component in the management of COPD, and multiple international guidelines describe the benefits of a pulmonary rehabilitation program (PRP). ${ }^{1-4}$ Short-term benefits include enhanced health-related quality of life (HRQoL) and self-efficacy, improved exercise performance and mood, reduced breathlessness, and reduced health care utilization. ${ }^{1,2}$ However, long-term benefits are less well preserved, and most measures of improvement return to baseline by $12-24$ months. ${ }^{5}$ Many attempts to prolong this effect have been trialed through a variety of maintenance and repeat programs with varied success. Many PRPs accept patients who would like to repeat the program, which is supported by recent guidelines. However, there is limited evidence to provide guidance as to the ideal interval between repeating the program, the indications to repeat, the populations who benefit most, or whether this is indeed of any benefit. ${ }^{6-9}$

Therefore, our question was: what is the magnitude of exercise capacity and quality of life improvements following PRP in patients with COPD who complete up to three PRPs based on clinical necessity for up to a period of 12 years?

\section{Methods}

\section{Patients}

We performed a retrospective chart review of all patients with COPD from our pulmonary rehabilitation database who completed a PRP on more than one occasion $(n=141)$ at two university hospital centers in New South Wales, Australia (Westmead and Mount Druitt Hospitals) between September 1, 2001 and September 1, 2013. All data were de-identified, and the study was approved by the Western Sydney Local Health District Human Research Ethics Committee (HREC2013/12/6.9(3898) QA) without further requirement for written informed consent.

\section{Data}

We included patients who had completed a minimum of two PRPs in the analysis. Each PRP consisted of an initial assessment, a minimum of 18 supervised exercise sessions, and a follow-up review, during the specified 12-year time frame. Specific measurements recorded at the beginning and end of each PRP included 6-minute walk distance (6MWD), HRQoL as measured by the St George's Respiratory Questionnaire (SGRQ),${ }^{10}$ mood as measured by the Hospital Anxiety and Depression Scale (HADS) ${ }^{11}$ and time interval between PRP sessions defined as the number of days from the last exercise session of the prior PRP to the first exercise session of the subsequent PRP. We also recorded anthropometric and demographic variables, and standardized measurements of resting lung function (forced expiratory volume in 1 second $\left[\mathrm{FEV}_{1}\right]$ and forced vital capacity) at the beginning and end of each PRP.

\section{Pulmonary rehabilitation program}

Adult patients with COPD with medically optimized symptomatic lung disease were referred by respiratory physicians or general practitioners and admitted to the outpatient PRP after an initial multidisciplinary assessment clinic with a respiratory or rehabilitation physician, nurse, and physiotherapist.

PRPs were completed at either Westmead or Mount Druitt Hospitals, and comprised 8-12 weeks of threetimes weekly supervised exercise sessions beginning with 20 minutes of ground walking, followed by a minimum of an additional 10 minutes of aerobic exercise of varying modalities (treadmill, cycle, or arm ergometer) dependent on patient needs and goals. A minimum of 20 minutes of upper and lower extremity strength training using free weight or body weight exercise was also completed according to patient needs and goals. We provided a weekly multidisciplinary group education session, covering topics according to published guidelines. ${ }^{1,2,12,13} \mathrm{We}$ encouraged patients to continue with their exercise program at home for the remaining 4 days of the week, and provided an individually prescribed home exercise program with an exercise diary during the PRP.

\section{Primary outcome measurement}

All 6-minute walk tests were performed on a level, enclosed, temperature-controlled corridor using standardized instructions. ${ }^{14}$ Two tests were performed at each of the prerehabilitation (initial clinic) and post-rehabilitation (review clinic) assessments at each PRP to account for a learning effect. ${ }^{15}$ The best 6MWD was recorded and used for analysis. An improvement of $30 \mathrm{~m}$ was regarded as the minimal clinical important difference (MCID). ${ }^{16}$ 6MWD was also expressed as a percentage of the healthy predicted value using reference equations which incorporated age, body mass index, and sex. ${ }^{17}$

\section{Secondary outcome measurements}

We assessed disease-specific quality of life using the Australian SGRQ validated for COPD,${ }^{10}$ with the MCID being $\geq 4$ units. ${ }^{18}$ We assessed mood with the HADS with scores greater than 7 units (out of a maximum of 21) in 
each of HADS-A or HADS-D indicating symptoms of anxiety or depression (respectively), ${ }^{11}$ and the MCID being a change $\geq 1.5$ units for total HADS in COPD. ${ }^{19}$

\section{Data analysis}

We used S-PLUS software version 8 (SolutionMetrics, Sydney, Australia) to analyze the longitudinal relationship between each PRP session and the outcome variables of 6MWD, SGRQ, and HADS using linear mixed models. We entered PRP session, 6MWD, HADS, and SGRQ into the model as fixed effects, and time between PRP sessions as a random effect. One-way analysis of variance with Tukey's post hoc analysis was used to compare the effects of different time intervals on 6MWD, SGRQ, and HADS between PRPs. These statistical analyses were performed using SPSS software (SPSS Version 23; SPSS, Chicago, IL, USA) and Prism 7 for Mac OS X (Version 7.0a; GraphPad Software Inc., San Diego, CA, USA). Statistical significance was accepted at $P<0.05$.

\section{Results}

\section{Baseline characteristics}

Data were available for 141 individuals (Table 1) who completed at least two PRPs (ie, an initial PRP and a repeat

Table I Patient demographics obtained immediately prior to commencing PRPI and prior to each subsequent program (PRP2 and PRP3)

\begin{tabular}{|c|c|c|c|}
\hline Variable & $\begin{array}{l}\text { PRP I } \\
(n=|4|)\end{array}$ & $\begin{array}{l}\text { PRP2 } \\
(n=|4|)\end{array}$ & $\begin{array}{l}\text { PRP3 } \\
(n=35)\end{array}$ \\
\hline Age (years) & $68 \pm 7$ & $7 I \pm 7$ & $72 \pm 7$ \\
\hline Male (\%) & 56 & 56 & 64 \\
\hline Home oxygen use (\%) & 15 & 19 & 25 \\
\hline BMI $\left(\mathrm{kg} / \mathrm{m}^{2}\right)$ & $26 \pm 5$ & $26 \pm 6$ & $25 \pm 5$ \\
\hline $\mathrm{FEV}_{1}(\mathrm{~L})$ & $1.02 \pm 0.42$ & $0.91 \pm 0.40^{*}$ & $0.84 \pm 0.38^{*, \#}$ \\
\hline $\mathrm{FEV}_{1}(\%$ predicted $)$ & $42 \pm 17$ & $39 \pm 16 *$ & $35 \pm 16 *$ \\
\hline FVC (\% predicted) & $80 \pm 20$ & $75 \pm 18$ & $77 \pm 19$ \\
\hline 6MWD (m) & $4 I I \pm 87$ & $373 \pm 106 *$ & $374 \pm 97 *$ \\
\hline SGRQ total (units) & $54 \pm 16$ & $56 \pm 14^{\S}$ & $56 \pm 10^{\S}$ \\
\hline HADS total (units) & $13(8-18)$ & $12(8-18)$ & $11.5(7-15)$ \\
\hline HADS anxiety (units) & $7(5-10)$ & $7(4-10)$ & $6(4-9.75)$ \\
\hline HADS depression (units) & $5(3-8)$ & $6(3.5-9)$ & $5(3-7)$ \\
\hline Current smokers (\%) & 10 & 8 & 2 \\
\hline Smoking (pack-years) & $43 \pm 23$ & $46 \pm 21$ & $41 \pm 19$ \\
\hline Time between PRP (months) & $\mathrm{N} / \mathrm{A}$ & $34 \pm 20$ & $29 \pm 15$ \\
\hline
\end{tabular}

Notes: Data are shown as mean \pm SD apart from HADS and its anxiety and depression domains, which are shown as median values (interquartile range). ${ }^{*} \mathrm{P}<0.000$ I vs $\mathrm{PRPI}$; ${ }^{\circledR P}<0.05$ vs $\mathrm{PRPI}$; ${ }^{\#} \mathrm{P}<0.00$ I vs $\mathrm{PRP} 2$; linear mixed effects. Abbreviations: $\mathrm{BMI}$, body mass index; $\mathrm{FEV}_{1}$, forced expiratory volume in I second; FVC, forced vital capacity; HADS, Hospital Anxiety and Depression Scale (0-22, 0= no symptoms of anxiety or depression); N/A, not available; PRP, pulmonary rehabilitation program; SD, standard deviation; SGRQ, St George's Respiratory Questionnaire (0-100 units, 0 units = best quality of life); 6MWD, 6-minute walk distance.
PRP). In addition, 35 individuals completed three PRPs, and 7 individuals completed a fourth PRP. Prior to PRP1, 56\% of the group comprised males, and mean body mass index was $26 \pm 5 \mathrm{~kg} / \mathrm{m}^{2}$.

Mean age of the 141 people who completed a PRP at least twice was $68 \pm 7$ years and mean $\mathrm{FEV}_{1}$ was $42 \% \pm 14 \%$ predicted (Table 1). There was a significant $\mathrm{FEV}_{1}$ decline from $1.02 \pm 0.42 \mathrm{~L}$ pre-PRP1 to $0.91 \pm 0.40 \mathrm{~L}$ pre-PRP2 $(P<0.0001)$, to $0.84 \pm 0.38 \mathrm{~L}$ pre-PRP3 (PRP2 vs PRP3; $P<0.001$ ). In addition, SGRQ increased over time between PRP1 and PRP2 (54 \pm 16 units vs 56 \pm 14 units; $P=0.04$ ) and between PRP1 and PRP3 (54 \pm 16 units vs 56 \pm 10 units; $P=0.01$; Table 1). 6MWD decreased between pre-PRP1 and pre-PRP2 (411 $187 \mathrm{~m}$ vs $373 \pm 106 \mathrm{~m} ; P<0.0001)$, but did not change between pre-PRP2 and pre-PRP3 (373 \pm 106 vs $374 \pm 97 \mathrm{~m} ; P>0.05)$. 6MWD expressed as \% predicted prior to PRP1, PRP2, and PRP3 was $84 \% \pm 19 \%, 79 \% \pm 23 \%$, and $80 \% \pm 24 \%$, respectively. HADS demonstrated no significant change over time.

\section{Improvements in 6MWD with each PRP}

The patients improved their 6MWD after each PRP, with a mean improvement of $58 \mathrm{~m}$ after PRP1 $(P<0.0001), 42 \mathrm{~m}$ after PRP2 $(P<0.0001)$, and $32 \mathrm{~m}$ after PRP3 $(P<0.003)$ (Figure 1). The improvement in 6MWD was less for PRP2 than for PRP1 $(P=0.03)$, but was not different for PRP3 compared to PRP1 $(P=0.15)$. The MCID of $30 \mathrm{~m}$ was achieved by $72 \%$ after PRP1, 63\% after PRP2, and 44\% after PRP3. For the 29 patients with complete 6MWD data who underwent PRP three times, total 6MWD expressed as \% predicted was

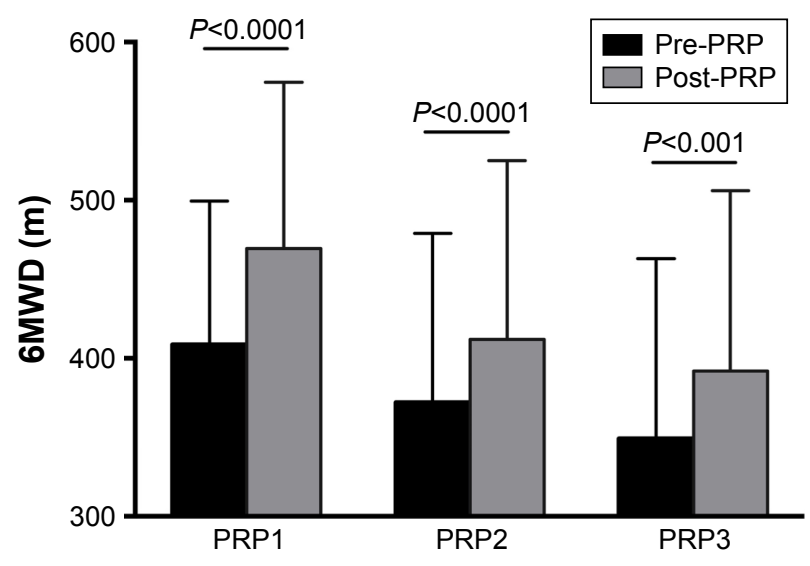

Figure I Grouped column graph of mean 6MWD pre-PRP (black) vs post-PRP (gray) for each PRP session.

Notes: $n=|4|$ for PRP sessions $I$ and 2 , and $n=35$ for session 3. Error bars represent standard deviations. Mean improvement was $58 \mathrm{~m}$ following PRPI, $42 \mathrm{~m}$ following PRP2, and $32 \mathrm{~m}$ following PRP3. All PRP sessions resulted in improvement in 6MWD $(P<0.0005$ for 6MWD post vs pre), although there was less improvement in PRP2 compared with PRPI $(P=0.03)$.

Abbreviations: PRP, pulmonary rehabilitation program; 6MWD, 6-minute walk distance. 
greater post-PRP1 vs post-PRP3 $(102 \% \pm 19 \%$ predicted vs $87 \% \pm 25 \%$ predicted; $P<0.0001)$.

\section{Improvements in health-related quality of life and mood with each PRP}

Mean SGRQ decreased after PRP1 by 7.0 units $(P<0.001)$ and after PRP2 by 4.9 units $(P<0.0001)$ but not significantly after PRP3 ( -3.2 units; $P=0.10$; Figure 2$)$. Improvements with PRP1 and PRP2 were greater than the MCID of 4 units, and the improvement at PRP2 was not significantly different from PRP1 $(P=0.13)$. Mean HADS improved after PRP1 with a decrease by 1.9 units $(P<0.0001)$ and after PRP2 by 1.7 units $(P=0.0001)$, but not after PRP3 ( $P=0.63$; Figure 3$)$. Improvements in HADS after PRP1 and PRP2 were greater than the MCID of 1.5 units and there was no difference between them $(P=0.76)$.

\section{Analysis by time interval between PRPs}

Fifty-eight patients returned for PRP2 within 2 years, 58 between 2 and 4 years, and 25 after 4 years (Table 2). The mean increase in 6MWD following repeat PRP was $47 \pm 66 \mathrm{~m}$ within 2 years, $38 \pm 45 \mathrm{~m}$ between 2 and 4 years, and $39 \pm 53 \mathrm{~m}$ after 4 years. There was no significant difference between change in 6MWD and the time interval between PRP1 and PRP2 $(P=0.7)$. There was an increased reduction in mean SGRQ at PRP2 for time interval $>4$ years vs $2-4$ years $(P=0.008)$. However, there was no significant difference between change in mean HADS and the time interval between PRP1 and PRP2 $(P=0.57)$.

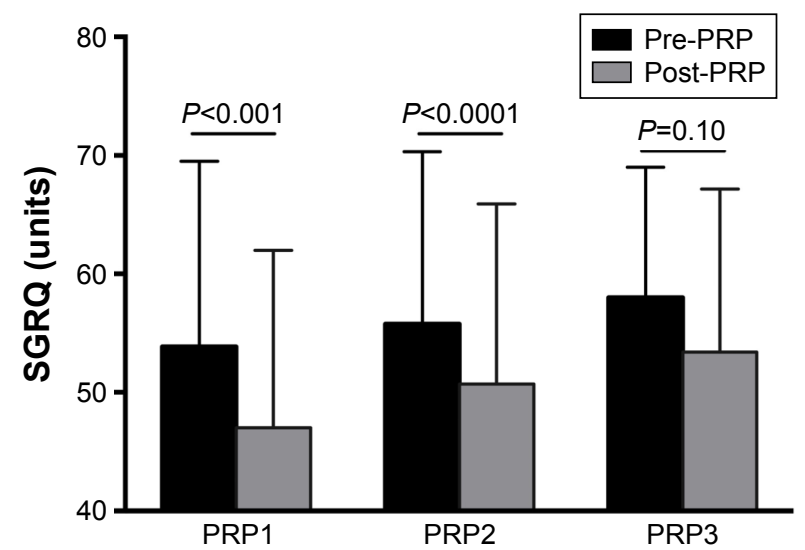

Figure 2 Grouped column graph of mean SGRQ pre-PRP (black) vs post-PRP (gray) for each PRP session.

Notes: Error bars represent standard deviations. Mean improvement was 7.0 units after PRPI and 4.9 units after PRP2. There were significant reductions in SGRQ following PRPI and PRP2 $(P<0.00 \mathrm{I})$ but not after PRP3 $(P=0.10)$.

Abbreviations: PRP, pulmonary rehabilitation program; SGRQ, St George's Respiratory Questionnaire.

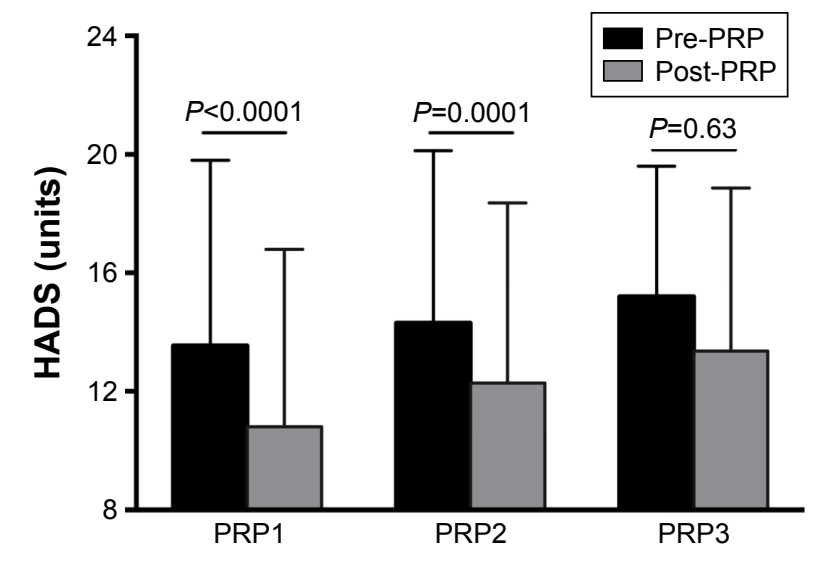

Figure 3 Grouped column graph of mean HADS pre-PRP (black) vs post-PRP (gray) for each PRP session.

Notes: Error bars represent standard deviations. Mean HADS improved more than the MCID only following PRPI and PRP2 ( -1.9 and -1.7 units, respectively; $P<0.00$ I; linear mixed effects) but not after PRP3 $(P=0.63)$.

Abbreviations: HADS, hospital anxiety and depression scale; MCID, minimal clinical important difference; PRP, pulmonary rehabilitation program.

\section{Discussion}

This study shows that there is a clinically meaningful improvement in functional exercise capacity after each repeat PRP episode despite an overall progressive decline in lung function. However, the magnitude of the improvement in exercise capacity was slightly less with repeat PRP compared with the initial PRP. HRQoL and mood improved following both a first and second PRP, but not after a third PRP. Furthermore, the time interval between initial PRP and repeat PRP did not affect the degree of improvement in functional exercise capacity.

Pulmonary rehabilitation in patients with COPD increases exercise capacity, decreases dyspnea, and improves HRQoL. Repeating pulmonary rehabilitation can be offered to patients as exercise benefits from a PRP diminish after $1-2$ years, ${ }^{5}$ but the ideal interval between repetition of a PRP remains

Table 2 Effect of interval between pulmonary rehabilitation programs on 6MWD and quality of life measures for 141 patients

\begin{tabular}{lllll}
\hline & \multicolumn{2}{l}{ Interval between PRPI and PRP2 } & P-value \\
\cline { 2 - 4 } & $\mathbf{0 - 2}$ years & $\mathbf{2 - 4}$ years & $>\mathbf{4}$ years & \\
\hline Number of subjects & 58 & 58 & 25 & \\
$\Delta 6 \mathrm{MWD}(\mathrm{m})$ & $47 \pm 66$ & $38 \pm 45$ & $39 \pm 53$ & 0.72 \\
$\Delta$ SGRQ (units) & $-6 \pm 1 \mathrm{I}$ & $-2 \pm 1 \mathrm{I}$ & $-10 \pm 13^{*}$ & 0.008 \\
$\Delta$ HADS (units) & $-2.0 \pm 6.1$ & $-1.2 \pm 4.8$ & $-2.4 \pm 5.1$ & 0.57 \\
\hline
\end{tabular}

Notes: Groups divided by time interval between first pulmonary rehabilitation program (PRPI) and repeat pulmonary rehabilitation program (PRP2), measured from the date of the last exercise session from PRPI to the first exercise session of PRP2. $P$-values represent one way ANOVA between groups. $* P=0.006$ for $>4$ years vs 2-4 years for SGRQ (one-way ANOVA, Tukey's post hoc analysis).

Abbreviations: ANOVA, analysis of variance; HADS, Hospital Anxiety and Depression Scale; PRP, pulmonary rehabilitation program; SGRQ, St George's Respiratory Questionnaire; 6MWD, 6-minute walk distance. 
unclear. ${ }^{1}$ Based on a study in patients with moderate COPD, there is likely little benefit to intervals less than 2 years; ${ }^{7}$ however, patients with severe COPD may benefit from a shorter interval. ${ }^{9}$ Current ATS, ERS and BTS pulmonary rehabilitation guidelines state that repeat PRP offers similar gains in exercise capacity and quality of life to the first $\mathrm{PRP}^{1,2}$ based on small observational studies ${ }^{8,20}$ and two small randomized controlled trials with 26 and 16 subjects, respectively. ${ }^{7,9}$ As home maintenance programs following a PRP appear to have benefit for only up to 2 years, ${ }^{21}$ repeating PRP when necessary may be indicated for long-term management of symptomatic patients with COPD.

Several studies have examined the effects of repeat pulmonary rehabilitation in patients with lung disease. , $7,9,20,22,23^{-1}$ However, there is significant heterogeneity in these studies particularly regarding study design and patient population, and there have only been three studies that have looked at the effects of a third PRP in patients with COPD. In controlled trials, adding a third PRP during a 2-year period ${ }^{7}$ or during a 12 -month period ${ }^{9}$ did not result in any additional improvement in 6MWD or HRQoL in the subjects compared with patients who only underwent two PRP over the same period. Our data differ from these controlled studies in that: 1) patients underwent repeat PRP based on physician-determined clinical necessity, rather than routine repeat PRP at set time intervals and 2) our lung function data and 6MWD data significantly declined over the time interval between PRP episodes reflecting "real-world" conditions, which were not seen in the controlled studies. In a 7-year observational study of 48 patients with moderate COPD who underwent five repeat PRPs, similar improvements were seen between the initial three PRPs spaced 12-18 months apart; however, improvements were lower than typically seen with PRPs and close to the MCID. ${ }^{8}$ In addition, over the 7-year time frame, the improvements in 6MWD with repeat PRP diminished, more closely reflecting the clinical time frame of our study. ${ }^{8}$

To our knowledge, we present the largest published cohort of patients with COPD undergoing a third PRP, which demonstrate the ongoing benefits of completing a repeat PRP. Although the reduced benefits of repeat PRP may be attributed to advancing airflow obstruction over time in our patients, recent studies have also demonstrated a reduced magnitude of improvement in 6MWD following a second program compared with the first program (approximately 2-4 years apart) despite no overall change in spirometric values. ${ }^{22,23}$ As our patients underwent repeat PRP over many years, it is also possible that advancing age itself may have contributed to an overall age-related decline in 6MWD. ${ }^{24}$
However, in the National Emphysema Treatment Trial, patients who had completed prior PRP had less improvement in 6MWD and HRQoL than those who underwent a PRP for the first time, although there was no difference in age or lung function, leading the authors to conclude that prior PRP appeared to have "blunted the response to pulmonary rehabilitation." ${ }^{25}$ Even after we expressed 6MWD as a percentage of predicted value, which factored in age-related decline, there was still a significant reduction in 6MWD after a third PRP compared with the initial PRP. This suggests that the reduction in 6MWD improvement after repeated PRP over time cannot be solely attributed to the effects of age. We speculate that the progression of airflow obstruction over time, increasing comorbidities in this population, ${ }^{26}$ increasing age,${ }^{24}$ and a learning effect from previous PRP, ${ }^{25}$ may all contribute to the outcome of repeat PRPs.

Although there was no significant improvement in mood after a third PRP, the HADS-A and HADS-D sub-domains in our cohort had median values all less than eight prior to each PRP, indicating that our group of COPD patients who completed the PRPs had overall relatively low levels of anxiety and depression (Table 1).

A strength of our study was the large cohort of patients that we analyzed, with a focus on only patients with COPD and not a population of mixed respiratory conditions. We also assessed the effects of repeat PRP not only on exercise capacity changes, but also on quality of life and mood, as the importance of patient-centered outcomes in COPD is being increasingly recognized. ${ }^{27}$ We used mixed-effects modeling, which is the ideal approach for analysis of multiple repeated measurements in each patient, factoring in time between PRPs as a random effect. ${ }^{28} \mathrm{~A}$ limitation of our study was that we did not have patient records between PRPs, including comorbidities, exacerbations, or hospitalizations. Although we have used 6MWD and patient-centered outcome measures to determine the efficacy of repeated PRPs, our database only looked at immediate outcomes of PRPs, and therefore we did not record other important long-term outcomes such as mortality, exacerbations of COPD, or the number of subsequent hospitalizations following PRP. In addition, as this study was not a randomized controlled trial, patient selection bias may have influenced our measured outcomes as we only studied patients who successfully completed repeat PRPs.

\section{Conclusion}

We conclude that there are clear benefits of repeat PRP for patients with $\mathrm{COPD}$, with improvements in functional 
exercise capacity following a third PRP. Furthermore, the time interval between repeat PRPs has no relation to the improvement in exercise capacity. We conclude that repeating PRPs based on clinical necessity for patients with COPD provides significant benefits, regardless of the duration of time between PRPs. Further research into whether a "blunted" response to repeat PRPs exists, and the long-term effects of repeat PRP on exacerbations and health care utilization, would help to further clarify the clinical benefits and the cost-benefit ratio of multiple repeats of a PRP.

\section{Acknowledgment}

The authors thank Dr Karen Byth and Ms Sharon Lee for assistance in statistical analysis.

\section{Disclosure}

The authors report no conflicts of interest in this work.

\section{References}

1. Spruit MA, Singh SJ, Garvey C, et al; ATS/ERS Task Force on Pulmonary Rehabilitation. An official American Thoracic Society/European Respiratory Society statement: key concepts and advances in pulmonary rehabilitation. Am J Respir Crit Care Med. 2013;188(8):e13-e64.

2. Bolton CE, Bevan-Smith EF, Blakey JD, et al; British Thoracic Society Pulmonary Rehabilitation Guideline Development Group; British Thoracic Society Standards of Care Committee. British Thoracic Society guideline on pulmonary rehabilitation in adults. Thorax. 2013;68 Suppl 2:ii1-ii30.

3. Marciniuk DD, Brooks D, Butcher S, et al; Canadian Thoracic Society COPD Committee Expert Working Group. Optimizing pulmonary rehabilitation in chronic obstructive pulmonary disease - practical issues: a Canadian Thoracic Society Clinical Practice Guideline. Can Respir J. 2010;17(4):159-168.

4. Ries AL, Bauldoff GS, Carlin BW, et al. Pulmonary rehabilitation: joint ACCP/AACVPR evidence-based clinical practice guidelines. Chest. 2007;131(5 Suppl):4S-42S.

5. Ochmann U, Jörres RA, Nowak D. Long-term efficacy of pulmonary rehabilitation: a state-of-the-art review. J Cardiopulm Rehabil Prev. 2012; 32(3):117-126.

6. Carr SJ, Hill K, Brooks D, Goldstein RS. Pulmonary rehabilitation after acute exacerbation of chronic obstructive pulmonary disease in patients who previously completed a pulmonary rehabilitation program. J Cardiopulm Rehabil Prev. 2009;29(5):318-324.

7. Foglio K, Bianchi L, Ambrosino N. Is it really useful to repeat outpatient pulmonary rehabilitation programs in patients with chronic airway obstruction? A 2-year controlled study. Chest. 2001;119(6): 1696-1704

8. Foglio K, Bianchi L, Bruletti G, et al. Seven-year time course of lung function, symptoms, health-related quality of life, and exercise tolerance in COPD patients undergoing pulmonary rehabilitation programs. Respir Med. 2007;101(9):1961-1970.

9. Romagnoli M, Dell'Orso D, Lorenzi C, et al. Repeated pulmonary rehabilitation in severe and disabled COPD patients. Respiration. 2006; 73(6):769-776.
10. Jones PW, Quirk FH, Baveystock CM, Littlejohns P. A self-complete measure of health status for chronic airflow limitation. The St. George's Respiratory Questionnaire. Am Rev Respir Dis. 1992;145(6): 1321-1327.

11. Zigmond AS, Snaith RP. The hospital anxiety and depression scale. Acta Psychiatr Scand. 1983;67(6):361-370.

12. Frith P. A Manual for Pulmonary Rehabilitation in Australia: Evidence Base and Standards. 2008. Available from: http://lungfoundation.com. au/wp-content/uploads/2014/02/rehab_standards_v3_31_july_2008. pdf. Accessed March 21, 2017.

13. Alison J. The Pulmonary Rehabilitation Toolkit on Behalf of The Australian Lung Foundation; 2009. Available from: http://www. pulmonaryrehab.com.au. Accessed March 21, 2017.

14. ATS Committee on Proficiency Standards for Clinical Pulmonary Function Laboratories. ATS statement: guidelines for the six-minute walk test. Am J Respir Crit Care Med. 2002;166(1):111-117.

15. Chandra D, Wise RA, Kulkarni HS, et al; NETT Research Group. Optimizing the 6-min walk test as a measure of exercise capacity in COPD. Chest. 2012;142(6):1545-1552.

16. Holland AE, Spruit MA, Troosters T, et al. An official European Respiratory Society/American Thoracic Society technical standard: field walking tests in chronic respiratory disease. Eur Respir J. 2014;44(6): 1428-1446.

17. Enright PL, Sherrill DL. Reference equations for the six-minute walk in healthy adults. Am J Respir Crit Care Med. 1998;158(5 Pt 1): 1384-1387.

18. Jones PW. Interpreting thresholds for a clinically significant change in health status in asthma and COPD. Eur Respir J. 2002;19(3): 398-404.

19. Puhan MA, Frey M, Buchi S, Schunemann HJ. The minimal important difference of the hospital anxiety and depression scale in patients with chronic obstructive pulmonary disease. Health Qual Life Outcomes. 2008;6:46.

20. Hill K, Bansal V, Brooks D, Goldstein RS. Repeat pulmonary rehabilitation programs confer similar increases in functional exercise capacity to initial programs. J Cardiopulm Rehabil Prev. 2008;28(6):410-414.

21. Güell MR, Cejudo P, Ortega F, et al. Benefits of long-term pulmonary rehabilitation maintenance program in severe COPD patients: 3 year follow-up. Am J Respir Crit Care Med. 2017;195(5):622-629.

22. Heng H, Lee AL, Holland AE. Repeating pulmonary rehabilitation: prevalence, predictors and outcomes. Respirology. 2014;19(7):999-1005.

23. Atabaki A, Fine J, Haggerty M, et al. Effectiveness of repeated courses of pulmonary rehabilitation on functional exercise capacity in patients with COPD. J Cardiopulm Rehabil Prev. 2015;35(4):272-277.

24. Casanova C, Celli BR, Barria P, et al; Six Minute Walk Distance Project (ALAT). The 6-min walk distance in healthy subjects: reference standards from seven countries. Eur Respir J. 2011;37(1):150-156.

25. Ries AL, Make BJ, Lee SM, et al; National Emphysema Treatment Trial Research Group. The effects of pulmonary rehabilitation in the national emphysema treatment trial. Chest. 2005;128(6):3799-3809.

26. Casanova C, Cote CG, Marin JM, et al. The 6-min walking distance: long-term follow up in patients with COPD. Eur Respir J. 2007;29(3): 535-540.

27. Celli BR, Decramer M, Wedzicha JA, et al; ATS/ERS Task Force for COPD Research. An official American Thoracic Society/European Respiratory Society statement: research questions in COPD. Eur Respir Rev. 2015;24(136):159-172.

28. Detry MA, Ma Y. Analyzing repeated measurements using mixed models. JAMA. 2016;315(4):407-408. 
International Journal of COPD

\section{Publish your work in this journal}

The International Journal of COPD is an international, peer-reviewed journal of therapeutics and pharmacology focusing on concise rapid reporting of clinical studies and reviews in COPD. Special focus is given to the pathophysiological processes underlying the disease, intervention programs, patient focused education, and self management protocols.

This journal is indexed on PubMed Central, MedLine and CAS. The manuscript management system is completely online and includes a very quick and fair peer-review system, which is all easy to use. Visit http://www.dovepress.com/testimonials.php to read real quotes from published authors 